\title{
More on Four-Dimensional Extremal Black Holes
}

\author{
N. Hambli 用用 \\ Physics Department, McGill University \\ 3600 University Street, Montréal, Québec, CANADA, H3A 2T8 \\ and \\ Laboratoire de physique nucléaire, Université de Montréal \\ CP 6128, Succ centre-ville; Montréal, Québec, CANADA, H3C 3J7
}

*E-mail address: hambli@hep.physics.mcgill.ca or hambli@lpsvsh.lps.umontreal.ca.

${ }^{\dagger}$ Address after September 1996: Institut des Hautes Études Scientifiques (IHES); 35 route de Chartres; F-91440 Bures-sur-Yvette, FRANCE. 


\begin{abstract}
We consider an embedding of the extremal four-dimensional Reissner-Nordström black hole into type $I I B$ string theory. The equivalent type $I I B$ configuration, in the D-brane weak-coupling picture, is a bound state of D1- and D5-branes threaded by fundamental type IIB strings. The bound state involves also a NSNS solitonic 5-brane, mimicking the role of the Kaluza-Klein magnetic monopole. The statistical entropy derived by counting the degeneracy of the BPS-saturated excitations of this bound state agrees perfectly with the (semiclassical) Bekenstein-Hawking formula.
\end{abstract}




\section{Introduction}

In the early seventies Hawking showed [1] using classical general relativity and quantum field theory in curved spacetime that black holes radiate thermally at a temperature $T_{H}=$ $\kappa / 2 \pi$, where $\kappa$ is the surface gravity. Since the presence of temperature always calls for an entropy the back hole acquires one which is equal to one quarter of the horizon area $[1,2]$. With this discovery, the earlier beautiful (but mere) analogy between the black hole classical mechanics and the laws of thermodynamics were put onto a much firmer foundation. Since thermodynamics is only a low-energy approximation to a more fundamental (microscopic) description, there ought to be a statistical interpretation of the black hole entropy in terms of its fundamental underlying quantum degrees of freedom. Over the past years, there have been different attempts to solve this problem and they all agree that a satisfactory and complete resolution of the problem requires a full quantum theory of gravity not just a semiclassical approximation [3-13].

Recently, there has been a considerable progress in this direction. This came through our improved understanding of the Ramond-Ramond (RR) charged string solitons through the Dirichlet brane (D-brane) description [14-20]. This rapid progress has led to a microscopic state counting of the black hole entropy in an explicit string computation using the D-brane technology [21].

The results of the D-brane counting of the black hole entropy were curiously all limited to five dimensions at the beginning [21-24]. The reason for this was that suitable fourdimensional black holes with non-zero horizon which are bound states of D-branes alone 
were not known円. One needs to add to the weak coupling bound state equivalent (of the semiclassical four-dimensional black holes) a Kaluza-Klein monopole like in the work of [28] or a symmetric 5-brane that carries an axion charge as performed in the work of [29]. The presence of the Kaluza-Klein monopole's magnetic charge (or the symmetric 5-brane's axion charge) will ensure that the four-dimensional string coupling is constant everywhere. This is a necessary condition for any D-brane counting of the Bekenstein-Hawking entropy to work $[25,30]$.

It is the purpose of our work here to study another example of these four-dimensional extremal black holes with non-vanishing horizon area and show, using the newly discovered D-brane technology, that its Bekenstein-Hawking entropy precisely matches the statistical entropy arising from the degeneracy of the BPS excitations (at weak coupling) of the corresponding D-brane bound state. Our four-dimensional extremal black hole is given by its supersymmetric embedding into a six-dimensional type $I I B$ string theory compactified on $K 3$. We find that the section $(t, r, \theta, \varphi)$ of the resulting black hole looks like a fourdimensional extremal Reissner-Nordström solution where the extra fifth $\left(x^{4}\right)$ and sixth $\left(x^{5}\right)$ coordinates are wrapping $\widehat{S}^{1}$ and $S^{1}$, respectively. In [28] two six-dimensional black hole examples were presented as uplifted four-dimensional extremal Reissner-Nordström charged black hole solutions of the Einstein-Maxwell gravity. One of the solutions solves

\footnotetext{
${ }^{1}$ The situation has changed since then. Recently, extremal four-dimensional black holes with finite area were constructed solely from intersecting D-branes of type II on $T^{6}$ [25]. These systems of D-branes can also be interpreted in terms of intersecting brane configurations of $\mathcal{M}$-theory [26]. In the latter case, one may use the 5-brane low-energy effective theory from quantization of the boundary states of the $\mathcal{M}$-theory two branes ending on the 5-branes to give the BPS state counting of the entropy [27].
} 
the background field equations of the type $I I A$ six-dimensional string theory on $K 3$. The other one is a solution of the type $I I B$ six-dimensional string theory on $K 3$. From the discussion of [28] one can use the $T$-duality transformation along the $x^{5}$ direction to map the type $I I A$ and type $I I B$ solutions problems to one another. The six-dimensional embedding that we deal with in the present calculation is a $T$-dualised version along the $x^{4}$ direction of the type $I I A$ solution of [28]. We find that the equivalent D-brane boundstate problem involves a solitonic NSNS 5-brane mimicking the role of the Kaluza-Klein monopole in stabilizing the horizon and making the four-dimensional dilaton (and hence the string coupling) constant.

In Section (2), we shall describe our six-dimensional solution as an uplifted fourdimensional extremal Reissner-Nordström black hole, and argue for its being supersymmetric. In Section (3), we compute the ADM mass, the horizon area and the Hawking temperature (which is zero at extremality). We also display the different RR and NSNS charges that are involved and express the entropy in terms of them. Section (4) deals with the statistical entropy from counting the BPS-saturated massless excitations of the of the weak coupling bound state which corresponds to the strong coupling extremal black hole configuration found in Section (2). The agreement with the Bekenstein-Hawking entropy is again remarkable. Our presentation ends in Section (5) by some concluding remarks and directions for future investigations. 


\section{The making of the extremal four-dimensional black hole}

The six-dimensional type $I I B$ superstring action including compactification on $K 3$ space is given in the string-frame metric byf

$$
S_{I I B}=8 V_{4} \int d^{6} x \sqrt{-g}\left[e^{-2 \phi}\left(R+4(\partial \phi)^{2}-(\partial \sigma)^{2}-\frac{1}{12} H^{2}\right)-\frac{e^{2 \sigma}}{12} F_{(3)}^{2}\right] .
$$

Apart from $F_{(3)}$, the field content of this type $I I B$ action is common to the other 6dimensional superstring theories, IIA on $K 3$ and heterotic on the four-Torus $T^{4}$. The field $\sigma$ is the modulus scalar for $K 3$ and $\phi$ is the six-dimensional dilaton. $H$ is the 3 -form field strength for the NSNS 2-form $B$, and $F_{(3)}$ is the 3 -form field strength for the RR 2-form $A_{(2)}$. The six-dimensional solution that solves the background field equations of action $S_{I I B}$ is given by:

$$
\begin{aligned}
d s^{2}= & -f^{-2} d t^{2}+f^{2}\left(d r^{2}+r^{2} d \theta^{2}+r^{2} \sin ^{2} \theta d \varphi^{2}\right) \\
& +\frac{1}{4 k^{2}}\left(d x^{4}\right)^{2}+\frac{1}{L^{2}}\left(d x^{5}\right)^{2} ; \\
A_{(2)}= & \frac{L}{\lambda \tilde{\lambda}} f^{-1} d t \wedge d x^{4}+\frac{2 k^{2}}{\lambda \tilde{\lambda}} \cos \theta d \varphi \wedge d x^{5} ; \\
B= & \frac{1}{L}\left(f^{-1}-1\right) d t \wedge d x^{5}+\frac{1}{2} \cos \theta d \varphi \wedge d x^{4} ; \\
e^{\phi}= & \frac{\lambda}{2 L k} ; \quad e^{\sigma}=\tilde{\lambda} ; \quad f=1+\frac{k}{r} .
\end{aligned}
$$

The $(t, r, \theta, \varphi)$ subspace of solution (2.2) yields the four-dimensional extremal ReissnerNordström black hole. The extra dimensions $x^{4}$ and $x^{5}$ are compactified on $\widehat{S}^{1}$ and $S^{1}$, respectively. It is also worth noting that the subspace $\left(\theta, \varphi, x^{4}\right.$ or $\left.x^{5}\right)$ has now the topology of $S^{2} \times\left(\widehat{S}^{1}\right.$ or $\left.S^{1}\right)$. This observation will in fact be useful later (in Section (3)) when we

\footnotetext{
${ }^{2}$ We could also consider compactification on $T^{4}$. We shall use the same notation as in [28] and units where $\alpha^{\prime}=1$.
} 
come to calculate the NSNS and RR charges generated by $B$ and $A_{(2)} 2$-forms as well as the Bekenstein-Hawking entropy. We also notice that the magnetic charge replacing the Kaluza-Klein monopole of $[28,29]$ is now given by the component $B_{\varphi 4}$ of the NSNS 2-form $B_{\mu \nu}$

Following the discussion of [28], the quick way to see that the solution in (2.2) is supersymmetric and preserves $1 / 4$ of the spacetime supersymmetries of the $N=2$ tendimensional theory is to find its ten-dimensional up-lift. However, one of the conclusions drawn from the investigation of [31] (and which are essential for our supersymmetry discussion) is the realization that the truncation of the type $I I B$ string theory to a ten-dimensional $N=1$ theory containing only its NSNS fields admits the extremal Reissner-Nordström black hole as solution. Most notably, this solution is supersymmetric and preserves $1 / 4$ of the $N=1, D=10$ theory, and is related to the ten-dimensional up-lift of (2.2) under the interchange of the RR and NSNS 2-forms. Furthermore, since in (2.1) the action $S_{I I B}$ has it 4-form potential set to zero the RR and NSNS 3-form field strengths should enter symmetrically in the supersymmetry transformations of the $N=1, D=10$ type $I I B$ theory [32]. Consequently, the ten-dimensional up-lift of the RR solution in (2.2) should mimic the NSNS Reissner-Nordström solution of [31], and preserves $1 / 4$ of the $N=1$, $D=10$ spacetime supersymmetries. The compactification to six-dimensions yields back the solution (2.2) which is then seen to preserve $1 / 4$ of the spacetime supersymmetries of the $N=2$ theory.

\footnotetext{
${ }^{3}$ These NSNS fields are the usual NSNS 2-form $B$ generating the 3 -form field strength $H$ and a tendimensional NSNS 2-form $A_{(2)}^{\prime}$ generating a 3-form field strength $F_{(3)}^{\prime}$ not to be confused with RR 2-form $A_{(2)}$ of 2.1).
} 
Next, we move to computing the different charges and thermodynamic quantities of the solution (2.2).

\section{Charges and thermodynamic quantities}

The extremal black hole solution in (2.2) can carry both electric and magnetic charges with respect to the RR 3-form field strength $F_{(3)}$ :

$$
\begin{aligned}
& Q_{1}=\frac{V_{4}}{2 \pi^{5 / 2}} \int_{S^{2} \times S^{1}} e^{2 \sigma *} F_{(3)}=\frac{2^{3}}{\sqrt{\pi}} V_{4} \frac{\tilde{\lambda}}{\lambda} k^{2} \\
& Q_{5}=2^{3} \pi^{3 / 2} \int_{S^{2} \times S^{1}} F_{(3)}=2^{7} \pi^{7 / 2} \frac{k^{2}}{\tilde{\lambda} \lambda}
\end{aligned}
$$

It carries also the following fundamental 'electric' and solitonic 'magnetic' charges with respect to the NSNS 3-form field strength $H$ :

$$
\begin{aligned}
& W_{F}=2^{4} \pi V_{4} \int_{S^{2} \times \widehat{S}^{1}} e^{-2 \phi *} H=2^{8} \pi^{3} V_{4} \frac{k^{2} L^{2}}{\lambda^{2}} ; \\
& W_{S}=\frac{1}{2^{2} \pi^{2}} \int_{S^{2} \times \widehat{S}^{1}} H=1 .
\end{aligned}
$$

The ADM mass of solution (2.2) ist

$$
M_{A D M}=2^{10} \pi^{3} V_{4} \frac{k^{2} L}{\lambda^{2}}
$$

The vanishing of $g_{t \mu}=0$ indicates that the solution carries no ADM momentum and the Hawking temperature is given by

$$
T_{H}=\frac{1}{2 \pi}\left|\frac{1}{f} \partial_{r} f^{-1}\right|_{\text {at the horizon }}
$$

which typically vanishes for the extremal black hole configuration of (2.2) at the horizon $r=0$. Using the six-dimensional metric and the knowledge that the topology of the horizon

\footnotetext{
${ }^{4}$ For the calculation of the ADM mass we have used the definition given in. [33].
} 
is $S^{2} \times \widehat{S}^{1} \times S^{1}$, the horizon area of the six-dimensional extremal black hole is then given by

$$
\mathcal{A}=2^{3} \pi^{3} \frac{k}{L}
$$

The Bekenstein-Hawking entropy is then simply

$$
S=\frac{\mathcal{A}}{\left(4 G_{N}\right)}=2^{10} \pi^{4} V_{4} \frac{k^{3} L}{\lambda^{2}}
$$

where we have used that the Newton's constant $1 / 4 G_{N}=2^{7} \pi V_{4} k^{2} L^{2} / \lambda^{2}$. In terms of the charges carried by the black hole the entropy $S$ is easily seen to take the much simpler form

$$
S=2 \pi \sqrt{Q_{1} Q_{5} W_{F} W_{S}}=2 \pi \sqrt{Q_{1} Q_{5} W_{F}} .
$$

This alternative way of expressing the Bekenstein-Hawking entropy will (in the next section) turn out to be useful when we come to compare it with the statistical entropy derived by using the D-brane counting method. A noteworthy feature also of equation (3.7) is that the solitonic charge enters trivially in the entropy since $W_{S}=1$. We shall appreciate more this point when we come to present the D-brane picture in Section (4).

\section{The statistical entropy from the D-brane picture}

In this section we describe the weak coupling bound state problem to which we map the semiclassical black hole field configuration (2.2). In the weak coupling problem, we compute the statistical entropy of a collection of D-branes and strings and reproduce the Bekenstein-Hawking formula (3.7). 


\subsection{The constituents of the weak coupling bound state}

Before dealing with the weak coupling problem, let us review the elements of the strong coupling problem at hand. From the discussion of Section (3), the strong coupling problem is an extremal black hole solution carrying $Q_{1}$ electric charge and $Q_{5}$ magnetic charge with respect to the RR 3-form field strength. This black hole exists also in the background field of macroscopic fundamental strings and a solitonic 5-brane carrying respectively the $W_{F}$ electric charge and unit $W_{S}$ of magnetic charge with respect to the NSNS 2-form $B$.

In turning to the description of the weak coupling equivalent configuration, we should recall that in the usual formulation of type $I I B$ string theory we find the $\mathrm{RR} p$-form field strengths, for $p=3,5,7$. Due now to the celebrated work of Polchinski [16], we understand these different RR $p$-forms as having as sources the D1-, D3- and D5-branes, respectively. In a $\sigma$-model description, we say that in ten dimensions the world volume of the Dp-branes couple to the type $I I B \mathrm{RR}(p+1)$-form potential $A_{(p+1)}$, and hence they carry electric charge with respect to the $\mathrm{RR}(p+2)$-form field strength $F_{(p+2)}$. Therefore, to identify the weak coupling D-brane bound state (to which we map the configuration (2.2)) one has simply to count the total number of charges from the RR fields in the solution (2.2). Since solution (2.2) involves also electric and magnetic $B$ charges, we are sure that the bound state is in the presence of fundamental type $I I B$ strings wrapping $x^{5}$ and a NSNS solitonic 5-brane wrapping $K 3 \times x^{5}$.

So based on what precedes, our weak coupling bound state is made of $Q_{1}$ D1-branes wrapping themselves around $\widehat{S}^{1}$ (or $x^{4}$ ) and carrying each a unit electric RR 3-form charge. Their partner in the D-brane bound state is $Q_{5}$ D5-branes which carry each a unit magnetic 
RR 3-form charge and wrap the entire $K 3 \times \widehat{S}^{1}$ space. The latter will in fact appear as strings in six dimensions, therefore forming a D-string composite with the $Q_{1}$ D1-branes?. Owing to the presence of the NSNS 2-form $B$, the complete picture of the bound state is a D-string composite threaded by fundamental type $I I B$ strings winding around $S^{1}$ and carrying the NSNS 3-form electric charge $W_{F}$ (which plays the role of a winding number). There is also the NSNS solitonic 5-brane carrying the unit $W_{S}$ magnetic charge with respect $H$ and winding around the whole of the internal space $K 3 \times S^{1}$, and hence overlap the $Q_{5}$ D5-branes along $K 3$. The BPS excitations of this weak bound state will preserve the supersymmetries of the $N=2$ theory as does its semiclassical strong coupling equivalent.

\subsection{The microscopic counting of entropy}

For the purpose of computing the entropy of the semiclassical extremal black hole, we shall now simply count the BPS-saturated excitations of the underlying bound state configuration just described [21]. Our counting method is actually similar to the description in $[29,34,35]$. The D-string composite at hand consists of $Q_{5}$ parallel D5-branes wrapping $K 3 \times \widehat{S}^{1}, Q_{1}$ parallel D1-branes wrapping $\widehat{S}^{1}$, and total $W_{F}$ winding number along $x^{5}\left(S^{1}\right)$, which is carried by the fundamental type $I I B$ strings. Let us consider the limit of large $W_{F}$ and $Q_{1}$. Since duality implies that entropy can only depend on the product $Q_{1} Q_{5}$ it

\footnotetext{
${ }^{5}$ The normalizations in (3.1) and (3.2) are defined in such a way that the charges $Q_{1}, Q_{5}, W_{F}$ and $W_{S}$ are naturally integer quantized. Thus the amount of each of the RR charges involved in (2.2) count the actual number of the D-branes entering in the bound state structure.

${ }^{6}$ The D-brane counting in the case where all the charges $Q_{1}, Q_{5}$ and $W_{F}$ are of the same order is discussed in $[36]$.

${ }^{7}$ The work of [18-20] explicitly checks this in some special cases.
} 
is sufficient to do the counting for the case $8 Q_{5}=1$. The $Q_{1}$ D1-branes are then marginally bound to the D5-brane but are free to wander within the transverse $K 3$ space. This motion is generated by $(1,1)$ Dirichlet open strings both of whose ends are stuck to the D1-branes. (The $(1,5)$ Dirichlet open strings do not contribute in our (particular $Q_{5}=1$ ) counting problem due to charge confinement $[29,34,35]$.$) \quad This yields 4 Q_{1} Q_{5}$ underlying winding energy microstates with minimum mass in boson-fermion pairs available within the Dstring composite which generate the transverse motion. The BPS-saturated excitations of the bound state are actually these $4 Q_{1} Q_{5}$ boson-fermion pairs counted as right-moving $(1,1)$ Dirichlet open strings (with no left-moving Dirichlet open strings) that end on the D1-branes and sharing the total winding number $W_{F}$ going around $x^{5}\left(S^{1}\right)$.

An alternative way to count these BPS-saturated string states is to picture the D1branes as instantons of the $U\left(Q_{5}\right)$ gauge theory on the worldvolume of the D5-brane [37,17]. More precisely, the D1-branes are the zero size limit of these instantons. In the work of [20], it was shown that the moduli space of one of these instantons (one D1-brane) corresponds to an $N=4$ superconformal field theory with $4 Q_{5}$ bosonic and an equal number of fermionic degrees of freedom. For a number of $Q_{1}$ D1-branes, the degrees of freedom of the resulting "instanton strings" are parametrized by $4 Q_{1} Q_{5}$ physical oscillators in boson-fermion pairs?. The logarithm of the maximum number of ways we distribute $W_{F}$

\footnotetext{
${ }^{8}$ For other values of $Q_{5}$ the counting problem of the BPS-saturated string states may be different but duality relates the resulting entropies. For instance if $Q_{5}>1$, see the discussion below which uses a counting method in which the bound state of a D1- and D5-branes is pictured as an instanton of the $U\left(Q_{5}\right)$ gauge theory on the D5-brane.

${ }^{9} \mathrm{~A}$ counting method along these lines was recently presented in a different but related context [38]. We are thankful to J. Maldacena for pointing this to us.
} 
amongst these string states gives the statistical entropy that we are after and which from the standard $(1+1)$-effective field theory on the string is given by the formula:

$$
S=\sqrt{\frac{\pi\left(2 N_{B}+N_{F}\right) E_{R} \mathcal{L}}{6}},
$$

where $N_{B}\left(N_{F}\right)$ is the number of species of right-moving bosons (fermions) with $E_{R}$ total winding energy $\left[0\right.$ in a box of length $\mathcal{L}$. Using $N_{B}=N_{F}=4 Q_{1} Q_{5}$ and $E_{R}=2 \pi W_{F} / \mathcal{L}$, we find (for the large $W_{F}$ thermodynamic limit)

$$
S_{\text {stat }}=2 \pi \sqrt{Q_{1} Q_{5} W_{F}}
$$

this is in prefect agreement with the Bekenstein-Hawking entropy of the semiclassical black hole (3.7).

Since in our problem (and from (3.2)) we have $W_{S}=1$, the D-brane counting presented in the preceding discussion is not affected by the presence of the single NSNS solitonic 5-brane. We should recall (in similarity with the $Q_{1}$ and $Q_{5} \mathrm{RR}$ charges) that the charge normalization in (3.2) are chosen in such a way that the charge $W_{S}$ count the actual number of the NSNS solitonic 5 -branes. Had we had $W_{S}>1$, we would have had these $W_{S} 5$-branes (which can be located anywhere on $\widehat{S}^{1}$ ) intersecting all the $Q_{1}$ D1-branes $\llbracket$. As a result, the $Q_{1}$ closed D1-branes break up into $\left(W_{S} Q_{1}\right)$ open 1-branes, each of which is bounded by a pair of the NSNS solitonic 5-branes. Hence, the winding number carried by the Dirichlet

\footnotetext{
${ }^{10}$ The winding energy is a 'stringy' property that does not exist in field theory. In order to wrap around the circle the string must be stretched, in so doing its energy increases with the winding number. This contribution is of course to be added to the usual one coming from the center-of-mass momentum.

${ }^{11}$ When $Q_{5}=1$ we have seen that the only relevant Dirichlet open strings for the counting of the degeneracy of the BPS-saturated excitations of the bound state are of $(1,1)$ type, whose ends are stuck on the $Q_{1}$ D1-branes.
} 
$(1,1)$ open strings will carry an additional label referring to the pair of the NSNS 5 -branes they lie in between [29]. The number of the underlying microstates in boson-fermion pairs becomes $N_{B}=N_{F}=4 Q_{1} Q_{5} W_{S}$. Inserting this into (4.1) with $E=2 \pi W_{F} / \mathcal{L}$ we obtain

$$
S_{\text {stat }}=2 \pi \sqrt{Q_{1} Q_{5} W_{F} W_{S}}
$$

in agreement with $(4.2)$ if $W_{S}=1$.

Finally, we recall that in our problem the NSNS solitonic 5-brane plays the role of the Kaluza-Klein monopole [28] in maintaining a constant modulus for the $x^{4}$ direction, and hence keeping the four-dimensional string coupling constant. A quantitative way to check this is to write down the equations of motion for the modulus field $\sigma$ and the dilaton $\phi$ from the the type $I I B$ action (2.1) after reformulating it in the Einstein-frame metric,

$g_{E \mu \nu}=e^{-\phi} g_{S \mu \nu}$. A glance at their equations of motion reveals that in order for $\sigma$ and $\phi$ to be constant one has to have the $B_{\varphi 4}$ component of the NSNS $B$-field, which yields the magnetic charge $W_{S}$ carried by the NSNS solitonic 5-branes.

\section{Concluding remarks}

We have displayed a new example of embedding the four-dimensional extremal ReissnerNordström black hole into type $I I B$ string theory. The solution carries both electric and magnetic charges with respect to the RR 3-form field strength $F_{(3)}$ and the NSNS 3-form field strength $H$. The corresponding weak coupling D-brane bound state involves D1branes winding around $x^{4}$ along with D5-branes wrapping $K 3 \times \widehat{S}^{1}$. The resulting D-string composite is threaded by fundamental type $I I B$ strings winding around $x^{5}$. There is also a NSNS solitonic 5-brane wrapping $K^{3} \times S^{1}$ whose role is to replace the Kaluza-Klein 
monopole in maintaining the $\phi$ and $\sigma$ fields constant.

We have also given an explicit derivation of the statistical entropy of the underlying weak coupling bound state configuration by counting the number of its BPS-saturated excitations (arising as Dirichlet low-energy open string states stuck on the D-branes). By exploiting the virtues of an $N=4$ or $N=8$ supersymmetric theory, one is able to extrapolate the result of the weak coupling limit using the D-brane picture back to the strong coupling regime. It is satisfying to find that the D-brane calculation gives an entropy which continues to match perfectly well the semiclassical Bekenstein-Hawking formula.

It would be interesting to study more four-dimensional black holes, as they would potentially shed more light on the counting problem of the statistical entropy from the D-brane bound states in string theory. A new class of background solutions can be found, for example, by using a combined $S T$ duality in six dimensions [32] to map type $I I B$ on $K 3$ to heterotic string theory on $T^{4}$, then performing a standard $O(22,6)$ rotation and using the $S T$ duality afterwards to go back to type $I I B$ on $K 3$ string theory. An interesting calculation would be then to find the weak coupling D-brane bound state equivalents to the new backgrounds thus obtained, and provide for each example the counting of the microscopic states responsible for the statistical entropy. This type of investigation would hopefully help in providing a more general approach to the entropy problem, which covers any four-dimensional black hole background solution.

At the end, may be, we should point out again that we have been restricted so far only to extreme and slightly near-extreme black hole configurations. This arises since in the present stage of the D-brane technology one can only count states at weak coupling, 
whereas black holes only exist at strong coupling. There appears to be no justification why should a weakly coupled description continue to hold far from extremality. However, most recently it was shown that there is a sense in which even black holes far away from extremality can be mapped into a composite of weakly interacting fundamental strings and D-branes $[2]$. This question was addressed in [34] for the five-dimensional black holes and generalized to the four-dimensional case in [35]. It would certainly be interesting to extend their work to other four-dimensional examples and also see whether the symmetry of the resulting statistical entropy continues to be consistent with $U$-duality symmetry.

\section{Acknowledgments}

First I would like to thank the Laboratoire de Physique Nucléaire (Université de Montréal) for their hospitality while this research was carried out. It is a pleasure to express all my gratitude to R. T. Sharp for his continuous support, help and encouragement. Finally, I would like also to thank R. C. Myers, J. Maldacena, R. R. Khuri, M. Paranjape and R. T. Sharp for useful discussions. This research was supported by NSERC of Canada and Fonds FCAR du Québec.

\section{References}

[1]. S. Hawking, Nature 248 (1974) 30;

S. Hawking Comm. Math. Phys. 43 (1975) 199.

\footnotetext{
${ }^{12}$ There have been earlier indications in [22] that the counting of string states at weak coupling agrees with the black hole entropy even in situations where one could not justify the extrapolation to strong coupling.
} 
[2]. J. Bekenstein, Lett. Nuov. Cimento 4 (1972) 737;

S. Hawking Phys. Rev. D7 (1973) 2333;

S. Hawking Phys. Rev. D9 (1974) 3292.

[3]. J. Bekenstein, Phys. Rev. D12 (1975) 3077.

[4]. S. Hawking, Phys. Rev D13 (1976) 191.

[5]. W. Zurek and K. Thorne, Phys. Rev. Lett. 54 (1985) 2171.

[6]. G. 't Hooft, Nucl. Phys. B335 (1990) 138;

G. 't Hooft Phys. Scr. T36 (1991) 247.

[7]. L. Susskind, hep-th/9309145.

[8]. L. Susskind and J. Uglum, Phys. Rev. D50 (1994) 2700 hep-th/9401070.

[9]. C. Teitelboim, hep-th/9510180.

[10]. A. Sen, Mod. Phys. Lett. A10 (1995) 2081 hep-th/9504147.

[11]. S. Carlip, gr-qc/9509024.

[12]. F. Larsen and F. Wilkczek, hep-th/9511064.

[13]. M. Cvetic and A. Tseytlin, hep-th/9512031.

[14]. J. Dai, R. G. Leigh and J. Polchinski, Mod. Phys. Lett. A4 (1989) 2073.

[15]. P. Horava, Phys. Lett. B231 (1989) 251.

[16]. J. Polchinski, Phys. Rev. Lett. 75 (1995) 4724 [hepth/9510017].

J. Polchinski, S. Chaudhuri and C. V. Johnson, hep-th/9602052.

[17]. E. Witten, hep-th/9510135. 
[18]. A. Sen, hep-th/9510229 and hep-th/9511026.

[19]. M. Bershadsky, V. Sadov and C. Vafa, hep-th/9511222.

[20]. C. Vafa, hep-th/9511088 and hep-th/9512078.

[21]. A. Strominger and C. Vafa, hep-th/9601029.

[22]. C. Callan and J. Maldacena, hep-th/9602043.

[23]. G. Horwitz and A. Strominger, hep-th/9602051.

[24]. J. C. Breckenridge, R. C. Myers, A. W. Peet and C. Vafa, hep-th/9602065;

J. C. Breckenridge, D. A. Lowe, R. C. Myers, A. W. Peet, A. Strominger and C. Vafa, hep-th/9603078

[25]. V. Balasubramanian and F. Larsen, hep-th/9604189.

[26]. I. R. Klebanov and A. A. Tseytlin, hep-th/9604166.

[27]. R. Dijkgraaf, E. Verlinde and H. Verlinde, hep-th/9603126.

R. Dijkgraaf, E. Verlinde and H. Verlinde, hep-th/9604055.

[28]. C. V. Johnson, R. R. Khuri and R. C. Myers, hep-th/9603061.

[29]. J. M. Maldacena and A. Strominger, hep-th/9603060.

[30]. F. Larsen and F. Wilczek, hep-th/9511064.

S. Ferrara and R. Kallosh, hep-th/9603090.

[31]. R. R. Khuri and T. Ortín, hep-th/9512177; hep-th/9512178.

[32]. E. Bergshoeff, C. M. Hull and T. Ortín, Nucl. Phys. B451 (1995) 547 [hepth/9504081; 
K. Behrndt, E. Bergshoeff and B. Janssen, hep-th/9512152.

[33]. J. X. Lu, Phys. Lett. B313 (1993) 29.

R. R. Khuri and R. C. Myers, hep-th/9512061.

[34]. G. T. Horwitz, J. M. Maldacena and A. Strominger, hep-th/9603109.

[35]. G. T. Horwitz, D. A. Lowe and J. M. Maldacena, hep-th 9603195.

[36]. J. Maldacena and L. Susskind, hep-th/9604042.

[37]. M. Douglas, hep-th/9512077.

[38]. J. Maldacena, hep-th/9605016. 